\title{
The characteristics of HIV serodiscordant couples consulted at the HIV Out-Patient Clinic in Warsaw
}

\author{
Joanna Grzeszczuk ${ }^{1}$, Agnieszka Wroblewska², Ewa Firląg-Burkacka², Justyna D. Kowalska ${ }^{2,3}$ \\ ${ }^{1}$ Medical University of Warsaw, Warsaw, Poland \\ ${ }^{2}$ HIV Out-Patient Clinic, Hospital for Infectious Diseases, Warsaw, Poland \\ ${ }^{3}$ Department of Adult's Infectious Diseases, Medical University of Warsaw, Warsaw, Poland
}

\begin{abstract}
Introduction: Human immunodeficiency virus (HIV) is predominantly transmitted through unprotected sexual intercourse. Condom use in long-term relations is uncertain, thus antiretroviral treatment can be the most efficient method of preventing HIV transmission. The objective of this study was to analyze epidemiological characteristics of HIV serodiscordant couples coming to consecutive visits.

Material and methods: Data on demographics, HIV viral load, hepatitis B virus (HBV) and hepatitis C virus (HCV) co-infections of HIV positive individuals (index patients) and data on sexual behavior, condom use in sexual relations were obtained from medical files.

Results: There were 52 couples investigated: 16 (31\%) homosexual and 36 (69\%) heterosexual ones. In general, 47 (90\%) HIV positive patients were on combination antiretroviral therapy (cART) and 42 (89\%) had HIV RNA below 50 copies/ml at last measurement. During one year of observation, 18 (35\%) patients had detectable HIV RNA. Out of index patients, 18 (35\%) were anti-HCV positive, 1 (2\%) was HBSAg positive and $4(7.7 \%)$ were VDRL positive. Twenty (38\%) persons admitted to have unprotected sexual contacts, 6 (29\%) homosexual men and $15(71 \%)$ heterosexual persons. Only 29 (56\%) HIV negative partners were tested regularly for HIV. Ten (19\%) of HIV positive patients already had children. There were five pregnancies during the time of the study. Four couples declared a desire to have children. Only 3 couples considered assisted reproduction.

Conclusions: Unprotected sex occurs often among serodiscordant couples observed. Thus regular cART should be emphasized. Frequent co-infections (HCV and syphilis) among index patients prove that it is necessary to test HIV negative partners for these infections. The observation confirms purposefulness of couple counseling about safe conception.
\end{abstract}

HIV AIDS Rev 2017; 16: 58-60 DOI: https://doi.org/10.5114/hivar.2017.66798

Key words: HIV, HCV, transmission, conception, serodiscordant couples.

\section{Introduction}

Despite the fact that effective combination antiretroviral therapy (cART) can prevent human immunodeficiency virus

Address for correspondence:

Joanna Grzeszczuk, Medical University of Warsaw, 37 Wolska St.,

01-201 Warsaw, Poland, e-mail: joogr@hotmail.com

(HIV) transmission, HIV epidemic is still on the raise in $\mathrm{Eu}-$ rope. In 2014, nearly 30000 persons were diagnosed with HIV in the European Union and European Economic Area with a rate of 6.4 cases $/ 100000$ people [1]. HIV epidemic in

Article history:

Received: 03.11.2016

Received in revised form: 03.01.2017

Accepted: 03.01.2017

Available online: 24.03 .2017
International Journal of HIV-Related Problems

HIV \& AIDS

R e vi e w 
this area is driven by sexual contacts with the highest proportion of new diagnoses reported among men who have sex with men (MSM) (42\%) [1]. The second most common transmission mode is through heterosexual contacts (33\%) [1]. The rate of new cases in Poland in 2014 increased by $4.4 \%$ in comparison to the previous year and elevated by $20 \%$ in comparison to median in 2008-2012 [2]. It is estimated by Joint United Nations Programme on HIV/AIDS (UNAIDS) and European Centre for Disease Prevention and Control (ECDC) that currently around 30000 people with HIV are living in Poland however those who are diagnosed and receive cART account for 9149 [3].

As a result of the widespread cART use, HIV infection is no longer perceived as an inevitably fatal diagnosis, but as a manageable chronic disease $[4,5]$. Longer survival, approaching to that seen in the general population, and improved quality of life lead to more HIV positive persons willing to be involved in stable relationships and having a longterm family plan [6-9]. Furthermore, effective treatment reduces significantly the risk of HIV transmission, which is another way to protect HIV negative persons from infection [10-14]. Accordingly, a systematic increase in the number of serodiscordant couples is observed. The exact number of people living in such relations in Poland remains unknown, and so is the exact number of people living with HIV.

The objective of this study was to analyze epidemiological characteristics of HIV serodiscordant couples treated in HIV Out-Patient Clinic, Hospital for Infectious Diseases in Warsaw. We aimed to observe current attitude to safe sex, procreation and efficacy of treatment in this group of patients.

\section{Material and methods}

The consecutive HIV positive persons attending the HIV Out-Patient Clinic in Warsaw in 2014 were asked about their partnership status and if they were in a relationship with an HIV negative partner, they were asked about sexual behaviors, condom use and family plans. Data on demographics, HIV viral load, hepatitis $B$, hepatitis $C$ and syphilis co-infections of HIV positive individuals were obtained from medical files.

\section{Results}

Fifty two couples were included in the study, $16(31 \%)$ homosexual and 36 (69\%) heterosexual ones. Among heterosexual couples there were 20 (56\%) HIV positive men and $16(44 \%) \mathrm{HIV}$ positive women. The median age of patients was 34 and it ranged from 19 to 66 years. In regard to treatment, 47 (90\%) HIV positive patients were on cART. Out of them, 42 (89\%) had HIV RNA below 50 copies/ml at last measurement. However, during one year of observation, 18 (35\%) patients had detectable HIV RNA, which ranged from 61 to 182292 copies/ml (median: 15037 copies/ml).

Regarding co-infections, 18 (35\%) HIV positive patients were anti-HCV positive, one was HBs antigen positive and four (7.7\%) were VDRL (Venereal Diseases Research Laboratory) positive (three homosexual men and one heterosexual man). Their partners were not tested for these infections.

Twenty (38\%) persons admitted to have unprotected sexual contacts, 6/16 (38\%) homosexual men and 14/36 (39\%) heterosexual persons, seven heterosexual men and seven heterosexual women. Only 29 (56\%) HIV negative partners were tested regularly.

In terms of procreation plans, ten (19\%) of HIV positive patients already had children, five men and five women living in heterosexual relationships. During the time of the study, there were five pregnancies, three in HIV positive women and two in HIV negative women. Four couples declared a desire to have children. Only three couples considered assisted reproduction (insemination or in vitro treatment) as a possible solution.

\section{Discussion}

There is a growing body of evidence that the most powerful tool to prevent HIV transmission is cART [13, 14]. According to current Polish and European guidelines, each HIV positive person should be treated immediately after diagnosis and regardless of CD4 count $[15,16]$. These trends are reflected well in our observation as in $2014,90 \%$ of participants were on cART and $89 \%$ of them had undetectable viral load at last measurement. The Landmark HPTN052 clinical trial showed $96 \%$ reduction of HIV infection among serodiscordant couples when the index patient was on successful cART. In meta-analysis of fifty observational studies, this reduction was $91 \%$ [12]. The PARTNER study proved no HIV infection among 1238 eligible couple-years of follow-up [14]. In our study, $38 \%$ of treated persons had viral load above the detection limit during one year of observation. There were no HIV transmissions reported in medical files, but only half of HIV negative partners were tested regularly. The reason why the remaining persons are not tested is unknown. As presented by the Danish study quoted above, the vast majority of HIV positive participants (85\%, $95 \mathrm{CI}$ : 81-89) did not disclose their HIV status [17]. This may further deepen the distress observed among serodiscordant couples, as presented in the study by Cheray and Jose [18].

One in three persons in our study were anti-HCV positive. In Poland the percentage of co-infection might reach up to $71 \%$ of HIV positive persons and was shown to reduce life expectancy by 17.3 years $[19,20]$. The HC EP09 and BINECO studies showed that HCV/HIV co-infected patients had HCV RNA found in the semen more frequently than those without HIV infection (37.8\% vs. 18.4\%) [21]. This may facilitate HCV permucosal transmission among HIV positive people. However, this finding was not confirmed by other studies [22].

In Poland, similarly to the whole Europe, HIV infections were mainly detected among people aged 20 to 39 years $(72.0 \%)[1,2]$. This highlights the necessity of counseling in the area of sexual and reproductive health $[16,17]$. It is therefore of great importance to educate HIV positive 
persons and their partners about the need for screening for other sexually transmitted diseases, such as HCV, HBV or syphilis. In addition, sexual partners of HBV positive persons should be vaccinated against HBV as an effective form of stopping HBV transmission.

Studies from North America and Europe presented that $20-33 \%$ of HIV-infected men and women indicate an intention to procreate [23-26]. The will of having children is often hindered by HIV infection [17]. A detailed Danish study revealed that almost half of the patients had children and $15 \%$ intended to conceive [17]. In our study nearly $20 \%$ of patients had children and four couples indicated reproductive wish.

Finally, neither prevention by HIV testing nor HIV treatment is feasible if people living with HIV are unaware of being infected and those who are aware remain outside specialist care. Both testing and linkage to care remains an area for improvement in Poland [27, 28].

\section{Conclusions}

Unprotected sex occurs often among serodiscordant couples in our study. Thus, early cART use and adherence to treatment should be emphasized. Frequent co-infections (HCV and syphilis) among HIV positive patients indicate necessity for testing HIV negative partners for sexually transmitted infections other than HIV. Furthermore, our observations show purposefulness of couple counseling about safe conception. Data presented in our study confirm that HIV positive persons on effective cART and in stable relationships are not posing a risk to their HIV negative partners and can safely conceive children.

\section{Conflict of interest}

The author's declared no potential conflicts of interest with respect to the research, authorship, and/or publication of this article.

\section{References}

1. European Centre for Disease Prevention and Control, Europe. WROf. HIV/AIDS surveillance in Europe 2014. Stockholm: ECDC. 2015.

2. Niedźwiedzka-Stadnik M, Pielacha M, Rosińska M. HIV and AIDS in Poland in 2014. Przegl Epidemiol 2016; 70: 249-259.

3. Krajowe Centrum ds. AIDS. Dane epidemiologiczne - Polska. Available at: http://www.aids.gov.pl/hiv_aids/450/. Accessed: 2016 October 26.

4. Mocroft A, Katlama C, Johnson AM, et al. AIDS across Europe, 1994-98: the EuroSIDA study. Lancet 2000; 356: 291-296.

5. Mocroft A, Phillips AN, Gatell J, et al. CD4 cell count and viral loadspecific rates of AIDS, non-AIDS and deaths according to current antiretroviral use. AIDS 2013; 27: 907-918.

6. Barreiro P, Duerr A, Beckerman K, et al. Reproductive options for HIV-serodiscordant couples. AIDS Rev 2006; 8: 158-170.

7. Bujan L, Daudin M, Pasquier C. Reproductive options for HIV-serodiscordant couples. Perspect Sex Reprod Health 2002; 34: 104.

8. Crankshaw TL, Matthews LT, Giddy J, et al. A conceptual framework for understanding HIV risk behavior in the context of supporting fertility goals among HIV-serodiscordant couples. Reprod Health Matters 2012; 20 (39 Suppl): 50-60.
9. Matthews LT, Mukherjee JS. Strategies for harm reduction among HIV-affected couples who want to conceive. AIDS Behav 2009; 13 Suppl 1: 5-11.

10. Anglemyer A, Horvath T, Rutherford G. Antiretroviral therapy for prevention of HIV transmission in HIV-discordant couples. JAMA 2013; 310: 1619-1620.

11. Baggaley RF, White RG, Boily MC. HIV transmission risk through anal intercourse: systematic review, meta-analysis and implications for HIV prevention. Int J Epidemiol 2010; 39: 1048-1063.

12. Baggaley RF, White RG, Hollingsworth TD, et al. Heterosexual HIV1 infectiousness and antiretroviral use: systematic review of prospective studies of discordant couples. Epidemiology 2013; 24: 110-121.

13. Cohen MS, Chen YQ, McCauley M, et al. Antiretroviral Therapy for the Prevention of HIV-1 Transmission. N Engl J Med 2016; 375: 830-839.

14. Rodger AJ, Cambiano V, Bruun T, et al. Sexual Activity Without Condoms and Risk of HIV Transmission in Serodifferent Couples When the HIV-Positive Partner Is Using Suppressive Antiretroviral Therapy. JAMA 2016; 316: 171-181.

15. Polish Scientific AIDS Society. Principles of Care for the HIV-infected Patients 2015. PTN AIDS, 2015.

16. SocietyEACS.Guidelinesv8.0.Availableat:http://www.eacsociety.org/ files/guidelines_8.0-english-revised_20160610.pdf. Accessed: 2016 2016 October 26.

17. Laursen T, Kesmodel US, Hojgaard A, et al. Reproductive patterns and fertility wishes among HIV-infected patients: survey from six outpatient clinics in Denmark. Int J Infect Dis 2013; 17: e851-856.

18. Cheray S, Jose JP. The determinants of distress among HIV discordant couples. HIV AIDS Rev 2015; 14: 119-125.

19. Grzeszczuk A, Wandalowicz AD, Jaroszewicz J, et al. Prevalence and Risk Factors of HCV/HIV Co-Infection and HCV Genotype Distribution in North-Eastern Poland. Hepat Mon 2015; 15: e27740.

20. Leszczyszyn-Pynka M, Ciejak P, Maciejewska K, et al. Hepatitis C coinfection adversely affects the life expectancy of people living with HIV in northwestern Poland. Arch Med Sci 2016; DOI: https://doi. org/10.5114/aoms.2016.58744.

21. Briat A, Dulioust E, Galimand J, et al. Hepatitis C virus in the semen of men coinfected with HIV-1: prevalence and origin. AIDS 2005; 19 : 1827-1835.

22. Bradshaw D, Lamoury F, Catlett B, et al. A comparison of seminal hepatitis $\mathrm{C}$ virus (HCV) RNA levels during recent and chronic $\mathrm{HCV}$ infection in HIV-infected and HIV-uninfected individuals. J Infect Dis 2015; 211: 736-743.

23. Chen JL, Philips KA, Kanouse DE, et al. Fertility desires and intentions of HIV-positive men and women. Fam Plann Perspect 2001; 33: 144-152, 165.

24. Ogilvie GS, Palepu A, Remple VP, et al. Fertility intentions of women of reproductive age living with HIV in British Columbia, Canada. AIDS 2007; 21 Suppl 1: S83-88.

25. Frodsham LC, Boag F, Barton S, et al. Human immunodeficiency virus infection and fertility care in the United Kingdom: demand and supply. Fertil Steril 2006; 85: 285-289.

26. Heard I, Sitta R, Lert F. Reproductive choice in men and women living with HIV: evidence from a large representative sample of outpatients attending French hospitals (ANRS-EN12-VESPA Study). AIDS 2007; 21 Suppl 1: S77-82.

27. Sullivan AK, Raben D, Reekie J, et al. Feasibility and effectiveness of indicator condition-guided testing for HIV: results from HIDES I (HIV indicator diseases across Europe study). PLoS One 2013; 8: e52845.

28. Ankiersztejn-Bartczak M, Firląg-Burkacka E, Czeszko-Paprocka H, et al. Factors responsible for incomplete linkage to care after HIV diagnosis: preliminary results from the Test and Keep in Care (TAK) project. HIV Med 2015; 16: 88-94. 